\title{
CAUSALIDAD Y EMERGENCIA
}

\author{
Santiago Ramón Castillo \\ Universidad de Santiago de Chile \\ Departamento de Filosofía \\ arqscr2017@gmail.com
}

RESUMEN: Se propone entender la relación mente-cuerpo partiendo del emergentismo, aunque esta propuesta no ha estado exenta de dificultades. Los esfuerzos del fisicalismo emergentista por encontrar poderes causales en lo mental han derivado en un eliminativismo de lo mental o a aceptarlo como fenómeno superveniente. En este artículo se intenta mostrar lo mental como un estadio emergente de lo físico pero sin pérdida de propiedades, pasando necesariamente por lo biológico como otro estadio de lo físico. Las propiedades mentales y biológicas, entonces, no se restringen a un ámbito separado del mundo físico, sino que constituyen formas diferentes de la fisicalidad pura.

PALABRAS CLAVE: mente-cuerpo, vida, fisicalismo, eliminativismo, monismo

SUMMARY: I propose to understand the mind-body relationship on the basis of emergentism, although this proposal has not been without difficulties. The efforts of emergentist physicalism to find mental causal powers have resulted in either an eliminativist stance regarding the mental or in its acceptance as a supervenient phenomenon. This article sets out to present the mental as an emerging stage of the physical but without any loss of properties, necessarily passing through the biological as another stage of physical. So mental and biological properties are not restricted to an area separate from the physical world, but represent different kinds of pure physicality.

KEY WORDS: mind-body, life, physicalism, eliminativism, monism

\section{Causalidad física, biológica y mental}

El problema de la relación de la mente con el mundo ha seguido un camino difícil después del descrédito del dualismo. Las diversas formas de monismo prevalecientes hoy en día se topan con serias dificultades para explicar cómo en una naturaleza enteramente física puede darse algo tan diferente de lo físico como las intenciones o los pensamientos. Tales dificultades han llevado a Kim a hablar de "la venganza de Descartes" (Kim 1998, p. 46).

No hay espacio aquí para referirse a los caminos emprendidos por el monismo, desde que apareció como propuesta hegemónica hace ya setenta años. Durante la primera mitad del siglo XX, el 
conductismo pareció ser un camino factible, pero los estudios en etología y neurofisiología lo debilitaron rápidamente. El conductismo negaba la realidad ontológica de la mente, con lo cual los estados mentales quedaban reducidos a disposiciones conductuales, lo que generó una fuerte crítica por su incapacidad explicativa. El rechazo al conductismo vino aparejado por dos teorías diferentes, la teoría de la identidad, o materialismo de la identidad mente-cuerpo, y posteriormente el funcionalismo. La teoría de la identidad concebía la mente como una realidad objetiva, pero la reducía a un mero funcionamiento biológico del cerebro. Por su parte, el funcionalismo distingue entre los componentes físicos del cerebro y la función de estos componentes; los estados mentales se convierten, entonces, en funciones cerebrales. El materialismo de la identidad mente-cuerpo (Place 1956, Smart 1962, Feigl 1967) fue derrocado rápidamente por el funcionalismo, propuesto en un inicio por Hilary Putnam (1960), quien mostró en forma contundente, con su argumento de la realizabilidad múltiple, que estados físicos diferentes pueden producir el mismo estado mental, con lo cual la correspondencia directa entre mundo y mente se rompe. El funcionalismo ha continuado buscando salidas a la relación mente-cuerpo hasta nuestros días, en manos de filósofos como Jerry Fodor (1983, 1987), David Lewis (1983) y Ned Block (1980).

Pero hay otro camino para intentar explicar la relación de los procesos mentales con el mundo, explorado mucho antes de los monismos de mediados del siglo pasado. Se trata del emergentismo, que nació a fines del siglo XIX, y se desarrolló con J.S. Mill (1906), Samuel Alexander (1920), C. Lloyd Morgan (1923), C.D. Broad (1925), y ha tenido cierto avance en nuestra época.

Es así como, en la actual filosofía de la mente la concepción de ésta como un emergente natural desde un estado menos complejo desempeñó un papel importante. La idea del emergentismo es que cuando una organización estructural alcanza cierto grado de complejidad puede manifestar propiedades por completo nuevas, que no estaban presentes en sus partes constituyentes. La mente, entonces, se aparece como candidata ideal para ser explicada como fenómeno emergente a partir de estados físicos complejos. Pero, por cierto, esta propuesta está lejos de presentarse exenta de dificultades. Es este camino, propuesto por el emergentismo, el que nos interesa explorar.

Las objeciones más fuertes en contra del emergentismo de lo mental provienen del fisicalismo. La diferencia entre ambas doctrinas radica en la comprensión de qué es metafísicamente básico. Lo característico del fisicalismo es afirmar que las propiedades mentales son 
propiedades físicas, o al menos proponer que están constituidas y son realizadas por propiedades físicas, o son supervenientes de ellas. Una de estas objeciones en contra de la mente como emergente de lo físico es la planteada por el eliminativismo. El planteamiento de rigor del eliminativismo respecto de los poderes causales de los estados mentales es el siguiente: si un suceso consistente en un estado intencional es un suceso físico/mental $c, \mathrm{y}$ al mismo tiempo se da un suceso $e$, consistente en la instanciación de ese estado intencional, ¿es en virtud de las propiedades mentales de $c$, o simplemente en virtud de las propiedades físicas de $c$ que es causado $e$ ? Es decir, $\Varangle c$ es eficaz qua físico o qua mental? Los eliminativistas dicen que basta con lo físico, lo mental sobra (Kim 1989, 1992, 1993, 2002; Denett 1969, 1981, Hofstadter y Dennett 1981).

Combatiremos esta postura intentando descubrir en qué consiste precisamente el fenómeno de la emergencia. Existe una corriente actual del emergentismo que se ha dado en llamar fisicalismo emergentista, de la cual Kim parece ser un buen representante. En esta doctrina se da, a nuestro juicio, la idea predominante de que ciertas entidades físicas adquieren, por añadidura, propiedades biológicas y mentales. El fisicalismo emergentista parece insinuar con esto que los átomos, las moléculas, o cualquier componente físico, conserva todas sus propiedades intactas al pasar de un estrato emergente a otro, con lo cual podría ser estudiado por la física, y más aún, podría tener todos los grados de libertad que posee cuando no está constreñido en un estado biológico o mental.

Tomando un conocido ejemplo de Fred Dretske (1988, p. 79) diremos que los fisicalistas quieren hacernos pensar que si la soprano rompe con su canto la copa de cristal, se trataría de un caso de causalidad física pura. Si el canto de la soprano daña el tímpano de un espectador, ello sería, asimismo, causalidad física. Si el mismo canto emociona a un espectador, se trataría de una afección neuronal y, en última instancia, física pura. Pretendemos, en cambio, que se trata de tres formas diferentes del ser de lo físico y, por lo tanto, de tres formas diferentes de causación, como intentaremos demostrar, o a lo menos hacer creíble. Para Dretske, aunque el canto de la soprano tiene propiedades mentales, en virtud de su letra, ello no cumple ningún papel en la rotura del cristal (lo cual es cierto), pero creemos que aquí hay una confusión respecto de los poderes causales.

Se convendrá que, aunque los tres niveles del ejemplo son físicos, son tres formas muy diferentes del ser de lo físico. ¿Qué los diferencia? Un átomo de hidrógeno libre (por ejemplo en el espacio sideral) 
es el mismo átomo que se sitúa en un tejido vivo, obedece las mismas leyes de la física en ambos casos; sin embargo, si lo observáramos (de ser ello posible) formando parte de un sistema (en nuestro ejemplo del tejido vivo), veríamos que él está constreñido en cuanto forma parte primero de moléculas, y a través de éstas, de células, que obedecen además leyes — tanto las moléculas como las células- que el átomo aislado "no conoce". El átomo del tejido vivo contribuye en la organización de un estrato superior (emergente) gracias a las vinculaciones que adquiere (llamaremos luego a estos vínculos "articulaciones"). Pese a que el átomo de nuestro ejemplo es el mismo, empieza a desempeñar un papel diferente según el complejo físico en que se sitúa. ¿Ha sido este hecho suficientemente analizado? En ese constreñimiento parece estar la clave de un comportamiento diferente. ¿Podemos decir que la célula posee un comportamiento físico? Sin duda que sí, pero el comportamiento complejo que presentan los átomos de esa célula ya no podemos llamarlo puramente físico. Los átomos de la célula y la célula misma como totalidad responden a leyes físicas, pero hay también en la célula otras leyes que ya no nos atrevemos a llamar físicas, y que son clasificables mucho mejor como "biológicas"; pero estas leyes, por decirlo de un modo gráfico, "albergan" en leyes físicas. ¿Qué sentido le damos a esta metáfora de "albergar"? De entrada diremos, siguiendo a Morgan, que lo biológico no se puede conformar sin lo físico:

[a]sí, para la evolución emergente, los sucesos conscientes en el nivel $C$ (de la mente) envuelven sucesos fisiológicos específicos en el nivel $B$ (de la vida), y éstos envuelven sucesos fisicoquímicos específicos en el nivel $A$ (de la materia). No puede haber algún $C \sin B$, ni un $B \sin A$. Ninguna mente sin vida; ni vida sin una base física. (Morgan 1923; la traducción es mía.)

Añadamos que los sucesos de un nivel dado son necesarios para los niveles superiores, pero no suficientes para su explicación. Como mostraremos más adelante, la existencia del estrato anterior es necesaria pero no suficiente debido a que en el proceso emergente aparece una dinámica nueva. En el estrato superior no se vulnera ley alguna del estrato inferior, pero esas leyes no pueden describir la realidad del nivel superior, donde han emergido más mundos posibles. No pretenderemos explicar ese proceso emergente, sin duda aún no explorado suficientemente, sino sólo describirlo. Lo vivo vive en lo físico; ese es su hábitat. De allí que decimos que lo biológico alberga en lo físico $o$, si vemos la metáfora desde otro punto de vista, lo físico hospeda 
lo biológico. Propondremos, también, que hay un paralelo exacto a esta relación entre los estratos siguientes, es decir, entre lo biológico y lo mental.

Todo esto nos lleva a una conclusión que parece bastante natural: no es posible separar el comportamiento físico del biológico en los seres vivos sin pérdida de la identidad del ser vivo. Hay, en el ser vivo, una indisolubilidad entre lo físico y lo biológico. No se trata de una mezcla de dos componentes, como podría ser la aleación de dos metales, sino la aparición de una entidad nueva que se mueve en dos planos a la vez: el físico y el biológico. No tenemos, entonces, derecho a separar drásticamente lo físico de lo biológico; y, extrapolando a un plano superior, lo físico de lo mental, o lo biológico de lo mental. Si lo biológico se mueve en dos planos, lo mental lo hace en tres.

Por ello, las separaciones entre lo físico, lo biológico y lo mental han sido siempre demasiado drásticas. El simple hecho de nombrarlas por separado rigidiza de por sí la escisión. Es cierto que los tres conceptos se formaron espontáneamente como respuesta a una fenomenología que se presenta muy diferente entre uno y los otros, que lleva a concebirlos como tres formas separadas del ser. Pero toda conceptualización es siempre drástica: el nombrar separa de un entorno, entorno que, sin embargo, contribuye inevitablemente a definir lo nombrado. El fruto no se entendería sin el árbol, o el humano sin su especie. Un objeto aislado, separado de su entorno, es un objeto "extraterrestre", incomprensible.

Pero más allá de esta defensa de una visión holística del mundo, nuestra intención es mostrar que la separación entre lo físico, lo biológico y lo mental ha sido arbitraria. Cuando en el lenguaje filosófico se habla de entidades biológicas o de entidades mentales sin más, se genera un malentendido. No existen entidades biológicas puras, ni entidades mentales puras.

Seguimos con estas ideas los planteamientos de mente corporizada, iniciados por Maurice Merleau-Ponty, quien aboga por una subjetividad situada: "El mundo y el cuerpo ontológicos que encontramos en la mismísima médula del sujeto no son el mundo en idea o el cuerpo en idea, es el mismo mundo contraído en punto de presa global, es el mismo cuerpo como cuerpo-cognoscente" (Merleau-Ponty 1985, p. 417).

Esta corriente ha continuado desarrollándose en el enactivismo. ${ }^{1}$ Por ello, volviendo al argumento fisicalista con que iniciamos esta 1999.

${ }^{1}$ Véanse especialmente Varela, Thompson y Rosch 1992, y Lakoff y Johnson 
exposición, lo modificaremos diciendo que si un suceso consistente en un estado intencional es un suceso físico/biológico/mental $c$, y al mismo tiempo se da un suceso $e$, consistente en la instanciación de ese estado intencional, $e$ es causado en virtud de las propiedades mentales de $c$, que albergan en las propiedades biológicas de $c$, que albergan en las propiedades físicas de $c$; es decir, $c$ es eficaz qua mental a través de lo físico y lo biológico.

Pero, desde el punto de vista que hemos bosquejado, las propiedades adquieren un sentido especial. Un mismo proceso posee muy diferentes propiedades según el estrato en el que se lo considere, y los efectos de esas propiedades son diferentes. Volviendo al ejemplo del canto: en el nivel físico, éste posee propiedades medibles con instrumentos (intensidad, frecuencia, etc.); en el ámbito físico-biológico, se trata de transformaciones de nivel somático, fenómeno algo más difícil de determinar (está aquí toda la respuesta del organismo vivo al sonido); en el nivel físico-biológico-mental, el proceso del ejemplo puede generar dolor, o placer, para lo cual no se ha descubiertoinventado una forma de medición.

Como una forma de superar los problemas terminológicos que hemos planteado - que separan, a nuestro juicio demasiado drásticamente, lo físico, lo biológico y lo mental- hablaremos de tres estados: físico puro, físico-biológico y físico-biológico-mental, que para abreviar llamaremos $f_{1}, f_{2}$ y $f_{3}$ respectivamente.

Siguiendo las ideas ya expresadas, diremos que las propiedades mentales no se restringen a un ámbito separado del cuerpo y del mundo físico. Debemos afirmar — como decía—, que ellas son mentales, biológicas y físicas a la vez. Lo que aquí se está rechazando es que exista una sola forma de ser de lo físico. Como dijimos, lo físico puro en lo $f_{2}$ y en lo $f_{3}$ está constreñido por un conjunto de articulaciones, que disminuyen considerablemente su "movilidad".

A falta de una mejor denominación, nos estamos refiriendo a tres estados de una misma entidad, lo físico, que conforman tres tipos de estratos diferentes. Intentaremos mostrar que los tres tipos de entidades físicas que hemos propuesto tienen poderes causales. Lo $f_{2}$ y lo $f_{3}$ tienen poderes no en cuanto entidades puramente físicas, sino físico-biológicas y físico-biológico-mentales, respectivamente.

No es que lo $f_{3}$ supervenga en lo $f_{1}$, ya que lo $f_{3}$ no está separado de lo $f_{1}$. No es que se dé primero un estado $f_{1}$ y luego un estado $f_{3}$, sino que todo proceso está ínsito en lo $f_{1}$, lo $f_{2}$ o lo $f_{3}$. Los procesos $f_{3}$ son de naturaleza físico-biológico-mental, y no están ligados necesariamente a procesos físicos puros. Así, lo $f_{2}$ puede ser 
afectado por lo $f_{1}$, como las células vivas se ven afectadas, por poner un caso cualquiera, por los rayos $\mathrm{X}$, pero no hay una relación de superveniencia entre los rayos $\mathrm{X}$, puramente físicos, y los procesos celulares, que son físico-biológicos. En otros términos, cada tipo de estado emergente posee autonomía respecto de los restantes.

Así, no tiene sentido decir que existen sucesos físicos causados por sucesos no físicos. En realidad, hay sucesos $f_{1}$ causados por sucesos $f_{2}$ o $f_{3}$, como también podemos decir que hay sucesos $f_{2}$ y $f_{3}$ causados por sucesos $f_{1}$.

Por lo tanto, debemos descartar la suposición del cierre causal del mundo físico. Esta suposición dice: "Cualquier suceso físico que tiene una causa en el momento $t$ tiene una causa física en $t$ " (Kim 1989; la traducción es mía). Debemos decir, en cambio, que cualquier suceso físico $f_{1}$ que tiene una causa en el momento $t$ tiene una causa ya sea $f_{1}, f_{2}$ o $f_{3}$ en $t$. Los poderes causales son diferentes en los tres casos.

Consecuentemente con lo expresado, debemos distinguir varios tipos de relaciones causales entre los diferentes niveles emergentes. En los tres niveles (de grano grueso) que hemos destacado se dan las relaciones que aparecen en el siguiente cuadro:

\begin{tabular}{|c|c|c|c|c|}
\hline \multirow{3}{*}{ Estado afectado } & \multicolumn{4}{|c|}{ Estrato de origen del proceso } \\
\cline { 2 - 5 } & & $f_{1}$ & $f_{2}$ & $f_{3}$ \\
\cline { 2 - 5 } & $f_{1}$ & 1 & $2^{\prime}$ & $3^{\prime}$ \\
\cline { 2 - 5 } & $f_{2}$ & 2 & 4 & $5^{\prime}$ \\
\cline { 2 - 5 } & $f_{3}$ & 3 & 5 & 6 \\
\hline
\end{tabular}

Lo físico puro $\left(f_{1}\right)$ que afecta a lo físico puro $\left(f_{1}\right)$ es 1 ; lo $f_{1}$ que afecta a lo físico-biológico $\left(f_{2}\right)$ es 2 , etc. Se puede observar que los estados 1,4 y 6 , que son las relaciones causales que se dan al interior de un mismo estrato, no son afectadas por la dirección del proceso causal; en cambio, los estados 2,3 y 5 , que corresponden a relaciones causales entre estratos diferentes, sí son diferentes según la dirección del poder causal, ya que es diferente, por ejemplo, lo $f_{1}$ que afecta a lo $f_{2}$, que lo $f_{2}$ que afecta a lo $f_{1}$. De allí que los designemos como parejas 2 y $2^{\prime}, 3$ y $3^{\prime}, 5$ y $5^{\prime}$. Por ejemplo, la soprano, que se encuentra en el estrato $f_{3}$ (asignarle otro estrato sería animalizarla o cosificarla), y que con sus notas rompe la copa de cristal (estrato $f_{1}$ ), está actuando en el nivel $3^{\prime}$; si daña el tímpano de un espectador, lo hace en el nivel 5'; si emociona a su auditorio, lo hace en el nivel 6. 
Resumiendo, podemos caracterizar los seis tipos de proceso del siguiente modo:

Tipo 1: De lo físico puro a lo físico puro. Allí se sitúa el mundo inanimado, dotado de lo que llamaremos más adelante movimiento puro. Es el campo que estudia la ciencia física.

Tipo 2. Es la afección de lo físico puro sobre la vida (sobre $f_{2}$ ). Es un amplio campo, cubierto por variadas ciencias especiales, como la biología.

Tipo $2^{\prime}$. Es la influencia de lo vivo $\left(f_{2}\right)$ sobre lo físico puro.

Tipo 3. Aquí se sitúa la afección de lo físico puro sobre los estados mentales (sobre lo $f_{3}$ ). Es la relación que va de lo físico puro al ser humano (o a ciertos animales). Está allí, por ejemplo, el asombro que produce la contemplación del cielo estrellado.

Tipo $3^{\prime}$. Es la acción de lo $f_{3}$ sobre lo físico puro. Allí se sitúa, por ejemplo, toda la industria del ser humano y la de muchos animales.

Tipo 4. Aquí se haya lo biológico $\left(f_{2}\right)$ que actúa sobre la vida misma. Por poner sólo un caso: la reproducción de los seres vivos.

Tipo 5. Es la influencia de lo vivo $\left(f_{2}\right)$ sobre lo mental $\left(f_{3}\right)$.

Tipo $5^{\prime}$. Es la acción de lo mental $\left(f_{3}\right)$ sobre lo biológico $\left(f_{2}\right)$.

Tipo 6. Aquí se sitúa la acción de lo mental sobre lo mental $\left(f_{3}\right.$ sobre $f_{3}$ ). Es el campo de la cultura en general, del pensamiento, del arte y de las ciencias humanas.

Esto no es obstáculo para que un mismo proceso o fenómeno esté presente en los tres estratos. Tomemos el caso del color: en el estrato $f_{1}$ es una radiación electromagnética de una frecuencia determinada. En $f_{2}$ es una afección de, principalmente, conos retinianos, y en general un estado neural determinado. En $f_{3}$ es la percepción de un color.

Otra característica que se desprende de lo propuesto es que todo estrato es precondición del siguiente. Por cierto, el estrato $f_{3}$ no existiría si no existiesen los estratos $f_{1}$ y $f_{2}$.

Una forma de avanzar en la demostración de esta propuesta es fijarse en que el recambio de sus partes no afecta a la identidad de una entidad (de un objeto). Por ejemplo, los seres vivos en los que el reemplazo de células por otras idénticas o muy similares se da en forma permanente sin afectar su identidad. Esto se puede visualizar también en un plano puramente físico: tomemos un motor, donde es posible (y necesario) el recambio de piezas sin afectar la totalidad del objeto. Por cierto, esta idea corresponde, en parte, a la propuesta por 
el funcionalismo. Nuestras diferencias con esta doctrina son de otro tipo: somos contrarios a la idea de "realización". Dicha "realización" implica la transmisión de poderes causales de un estrato a otro, con lo cual no concordamos. Los poderes causales no se transmiten sin cambios de un estrato emergente a otro. El poder causal del cloro ya no está presente en el cloruro de sodio; el cloro ha perdido sus poderes causales "en beneficio" del cloruro de sodio. El compuesto molecular se hospeda en el estrato inferior, y adquiere en cierto modo autonomía, sus poderes causales son ahora de naturaleza diferente. En esto consiste justamente la emergencia. Por ello no podemos decir que lo emergente es la realización del estrato inferior. Las propiedades mentales no son idénticas a las propiedades de estados cerebrales no por las razones que aduce el funcionalismo (es decir, por la realizabilidad múltiple), sino porque no hay herencia causal entre estratos. Los poderes causales cambian y, por lo tanto, cambian las propiedades.

\section{Dinámicas causales}

En nuestra propuesta es conveniente distinguir acción de movimiento. Esto resulta necesario si se quiere discernir dinámicas causales diferentes en los tres estratos. Llamamos "acción" al proceso que se inicia en el nivel $f_{3}$, "movimiento autónomo" al que se inicia en el nivel $f_{2}$, y "movimiento puro" a aquel que parte del nivel $f_{1}$.

Esto se puede graficar del siguiente modo:

\begin{tabular}{|l|l|c|c|c|}
\hline & \multicolumn{4}{|c|}{ Estrato } \\
\hline \multirow{4}{*}{ Dinámica causal } & & $f_{1}$ & $f_{2}$ & $f_{3}$ \\
\cline { 2 - 5 } & Movimiento puro & Sí & Sí & Sí \\
\cline { 2 - 5 } & Movimiento autónomo & No & Sí & Sí \\
\cline { 2 - 5 } & Acción & No & No & Sí \\
\hline
\end{tabular}

Lo que aquí se propone es que, en cada uno de los estratos emergentes $\left(f_{1}, f_{2}\right.$ y $\left.f_{3}\right)$, se produce una dinámica causal diferente. En el nivel físico-biológico, parecería que un motor inicia su marcha: lo que era enteramente condicionado encuentra autonomía. En el nivel físico-biológico-mental, por encima de un movimiento propio aparece la capacidad de dirigir ese movimiento.

Así, en lo $f_{1}$ puede darse sólo el movimiento puro; en lo $f_{2}$ el movimiento puro y el autónomo, y en lo $f_{3}$ pueden darse los tres tipos de 
dinámica. Cada estrato se apoya en el anterior. Lo mental $\left(f_{3}\right)$ no sería posible sin lo biológico $\left(f_{2}\right)$, que le aporta el movimiento autónomo, el cual es una dinámica típicamente biológica. Lo mental aprovecha parte del movimiento autónomo para hacer emerger la acción, que es la dinámica característica de lo mental. De forma parecida, lo biológico no sería posible sin lo físico, que le aporta el movimiento puro, que es una propiedad física pura. Lo biológico aprovecha parte del movimiento puro para hacer emerger el movimiento autónomo.

A manera de ejemplo, tomemos el paso de lo $f_{1}$ a lo $f_{2}$. Lo físico puro indudablemente tiene poderes causales sobre lo $f_{2}$, pero lo $f_{2}$ mismo dispone de poderes causales añadidos que no pueden ser considerados físicos puros. El vuelo que emprende un ave asustada por el granizo repentino tiene su origen en el fenómeno físico puro granizo, pero no podemos decir que el emprender el vuelo mismo sea causado, físicamente, por el granizo. El fenómeno físico, que pertenece al ámbito $f_{1}$, ha puesto en marcha otro fenómeno, $f_{2}$, que es el vuelo, pero el vuelo es contingente: podría darse o no, dependiendo de la autonomía del ser vivo. En lo $f_{2}$ hay una dinámica propia dada por el movimiento autónomo. La vida ha descubierto un motor que la mueve, que es parcialmente independiente del movimiento físico puro. Lo más característico de lo vivo es el movimiento autónomo; así como lo más propio de la muerte es el cese de ese tipo de movimiento.

La teoría que estamos proponiendo perecería generar una multiplicación innecesaria de los entes que pueblan el mundo, pero a nuestro juicio es justamente lo contrario. Se trata más bien de una simplificación, ya que en la actualidad se admiten por lo menos tres tipos de entidades bien diferenciadas: lo físico, lo biológico y lo mental, y todos los esfuerzos por reducir las dos últimas a la primera han resultado infructuosos. Un fisicalista convencido diría que se trata, en el fondo, de una sola cosa, pero se vería en aprietos al intentar fundamentar las diferencias. Lo que proponemos es un tipo de sustancia con diferentes propiedades y comportamientos.

Uno de los argumentos utilizados por los fisicalistas para defender la clausura causal del mundo físico es que, ya que a veces actuamos intencionalmente, es obvio que esas acciones generarían ciertos cambios físicos. Tal cosa constituiría una violación del principio de clausura causal, ya que habría una "intrusión" de causas no físicas en el ámbito físico. Luego, como lo dice Kim:

Abandonar este principio es admitir que no puede haber en principio ninguna teoría física completa de los fenómenos físicos; que la física teórica, en la medida en que aspire a ser una teoría completa, ha de 
dejar de ser física pura e invocar poderes causales irreductiblemente no físicos. (1993; la traducción es mía.)

Este argumento muestra cómo se ha instalado entre los fisicalistas una confusión categorial. ¿Por qué deberían interesarle a la física los fenómenos físico-biológicos o físico-biológico-mentales? Por ejemplo, ¿está dentro de su ámbito el crecimiento de las plantas? La clausura causal falla, a nuestro juicio, porque no hay una "intrusión" de causas "no físicas" en lo físico, ya que lo mental $\left(f_{3}\right)$ tiene un hábitat físico, como lo propone, entre otros Varela, con su idea de mente corporizada (Varela, Thompson y Rosch 1991). De este modo, lo mental adquiere legitimidad.

El hecho de que un mismo proceso físico puro $\left(f_{1}\right)$, o físicobiológico $\left(f_{2}\right)$, o físico-biológico-mental $\left(f_{3}\right)$ afecte de diversa forma cada estrato muestra que no sólo los procesos físicos puros $\left(f_{1}\right)$ determinan todos los hechos. En el ejemplo del canto, la ciencia física no puede explicar todas sus propiedades, porque la física del canto (la onda transmitida en un medio puramente físico) ha sido modulada por un organismo vivo (a través de los órganos de la fonación), que se basa en el movimiento autónomo, a lo cual la física es ciega; por otra parte, el canto ha sido modulado por una mente que se basa en el movimiento libre, es decir, en la acción, lo cual está aún más lejos de ser abarcado por las ciencias físicas.

En los estratos superiores, $f_{2}$ y $f_{3}$, pese a estar constituidos cuantitativamente por la misma materia, se dan formas diferentes. Entiendo la emergencia como una transformación. Lo vivo es materia transformada, y lo mental es esa materia transformada (viva), que experimenta una nueva transformación. No se trata aquí de una base material que adquiere una función, ni de algo que superviene de lo físico, que son soluciones que se resisten a abandonar por completo el dualismo. En cada proceso emergente aparecen nuevas dinámicas; lo vivo parece rebelarse contra una fisicalidad pasiva y adquiere una independencia que requiere nuevas ciencias para su comprensión; aparece una autonomía que las ciencias físicas no logran penetrar. En lo mental emerge una nueva forma de autonomía, más completa, que llamamos acción.

Kim hace ver que, en el modelo estratificado, propio de la emergencia, se da un problema crucial: cómo se relacionan las propiedades de un nivel dado con las de otro nivel. Por ejemplo, cómo se relacionan las propiedades puramente físicas de un organismo vivo con sus propiedades biológicas. "La respuesta clásica positivista - dice $\mathrm{Kim}$ - es que las propiedades distintivas de las entidades de un nivel 
dado son reducibles a las propiedades y relaciones que caracterizan a las entidades de los niveles inferiores, o explicables reductivamente en términos de ellas. En esto consiste el reduccionismo" (Kim 2014, p. 5; las cursivas son de Kim). Nuestra propuesta se inscribe dentro del no reduccionismo y consiste en que las propiedades de un nivel superior albergan en las de nivel inferior, en el sentido de que las utilizan para su propio desarrollo; las propiedades de lo vivo se asientan en lo físico puro, y las propiedades mentales en las propiedades de lo vivo.

No es posible explicar las propiedades de un estrato con las de otro inferior, porque las propiedades del superior han sido moldeadas (moduladas) con el material inferior, y por eso mismo las propiedades de esa modulación escapan al alcance de las ciencias que estudian las propiedades del estrato inferior. Queda, por cierto, la pregunta sin resolver de en qué consiste esa capacidad de moldear el material del estrato inferior; es decir, qué es esa autonomía emergente de la que hablamos.

¿Qué es, entonces, lo que distingue un estrato de otro? ¿Si los tres que hemos mencionado son tres formas diferentes del ser de lo físico, en qué consiste exactamente esa diferencia? Aquí hemos introducido un concepto que nos ayudará a describir esas diferencias: el concepto de modulación.

Como una forma de mostrar la íntima relación entre los conceptos de poder causal y modulación, volvemos al ejemplo del canto de la soprano. El poder causal del canto que rompe el cristal tiene la misma naturaleza del que mueve el tímpano del oyente y genera una transmisión neuronal de un estímulo. En este caso, lo físico puro $\left(f_{1}\right)$ se encuentra afectando lo $f_{2}$, pero se sitúa en una fisicalidad modulada. La transmisión del proceso causal transita ahora por rutas diferentes. Algo similar sucede con lo $f_{1}$ que afecta a lo $f_{3}$, y lo $f_{2}$ a lo $f_{3}$.

Como se desprende de los cuadros anteriores, está claro que aceptamos una causalidad descendente. En nuestro repetido ejemplo del canto, el sonido de éste viajando en el aire posee, aparentemente, sólo propiedades físicas; y, sin embargo, proviene de dos fuentes que lo han modulado: una mente y un organismo vivo. En cierto modo, ese sonido viene cargado, potenciado, por la modulación previa.

No es que lo $f_{2}$ o lo $f_{3}$ supervengan a lo $f_{1}$, sino que se trata de una transformación de lo físico puro. La idea de superveniencia, que es una de las más frecuentes en el actual panorama del fisicalismo no reductivo, se trasluce en el siguiente texto: "El fisicalismo es verdadero si y sólo si cada instanciación de propiedades o bien es 
física o bien es necesitada por alguna instanciación de una propiedad física" (Stoljar 2010, p. 37; la traducción es mía).

Lo que esta fórmula expresa es una dependencia de lo no físico de lo físico. Si entendemos, por ejemplo, los procesos mentales como no físicos, entonces habrá una dependencia, no necesariamente nomológica, de lo mental respecto de lo físico. Pero hay dos ideas subyacentes en esta fórmula que no podemos aceptar. Una es que existe una división tajante entre procesos físicos y no físicos. Está aquí la idea no bien expresada de que pueden darse procesos totalmente no físicos, aun cuando ellos sean dependientes de una base física, y ello es contrario a nuestra postura. Pese a las reiteradas protestas de muchos filósofos en contra del dualismo, se insiste en una separación tajante entre mente y cuerpo. Tanto es así que Kim ha llamado a este fenómeno "la venganza de Descartes" (2002, p. 46). La segunda idea encubierta en esta formulación es que los procesos físicos (a nuestro entender puros) son los únicos necesarios para la instanciación de los procesos "no físicos". Los fisicalistas no reduccionistas desean que exista una relación positiva entre los diversos niveles emergentes, pero no han encontrado algo diferente de la superveniencia y del funcionalismo, en la cual la primera no es una relación causal y la segunda lleva, de modo al parecer inevitable, a una desvalorización de lo mental.

En nuestra propuesta, lo $f_{1}$ no causa lo $f_{2}$, ni lo $f_{3}$. Debemos decir, en cambio, que lo $f_{2}$ aprovecha la dinámica de lo físico puro. Lo $f_{1}$ constituye el medio en que se desarrolla lo $f_{2}$, y a su vez lo $f_{2}$ el medio de lo $f_{3}$. Lo $f_{2}$ posee autonomía respecto de lo $f_{1}$, y lo $f_{3}$ es capaz de actuar por sobre lo $f_{2}$. En qué consisten estos poderes causales es algo que intentaremos desarrollar en trabajos posteriores.

Haremos notar que el fisicalismo ha intentado encontrar una relación causal que va de lo físico a lo mental, desconociendo en gran medida lo biológico. Hallar una vía causal de lo físico a lo no físico (mental) es un propósito infructuoso. Dado que lo $f_{3}$ es un todo unitario, lo $f_{1}$ sólo puede producir efecto sobre lo $f_{3}$ como totalidad y no sólo sobre su parte física pura. Por ello no tiene sentido buscar una reducción de lo $f_{3}$ a lo físico puro. Pero sí hay causalidad descendente desde lo $f_{2}$ a lo $f_{1}$ a través de la autonomía de lo biológico, y la hay de lo $f_{3}$ a lo $f_{2}$ o a lo $f_{1}$ a través de la acción.

Lo físico puro se encuentra en los tres estratos, aunque modulado en lo $f_{2}$ y $f_{3}$ de acuerdo con la naturaleza de estos niveles. Esta presencia permanente de lo físico puro permite la transmisión del poder causal, la cual es posible gracias a la presencia constante de lo físico puro. Y no es que, por ejemplo, lo mental inicie una acción 
para ser instanciada posteriormente por lo físico puro; se trata, más bien, de una acción como totalidad, en que la naturaleza de lo $f_{3}$, que es física, permite la efectividad en el mismo estrato o en otros.

\section{BIBLIOGRAFÍA}

Alexander, Samuel, 1920, Space, Time, and Deity, Macmillan, Londres.

Block, Ned, 1980, "What Is Functionalism?", Readings in Philosophy of Psychology, vol. 1, Harvard University Press, Cambridge, Mass., pp. 171-184.

Broad, C.D., 1925, The Mind and its Place in Nature, Kegan Paul, Trench and Trubner/Harcourt, Brace, Londres/Nueva York.

Dennett, Daniel, 1981, Brainstorms: Philosophical Essays on Mind and Psychology, MIT Press, Cambridge, Mass.

- 1969, Content and Consciousness, Routledge and Kegan Paul, Londres. [Versión en castellano: Contenido y conciencia, trad. José María Lebrón, Gedisa, Barcelona, 1996.]

Dretske, Frederick, 1988, Explaining Behavior: Reasons in a World of Causes, MIT Press, Cambridge, Mass.

Feigl, Herbert, 1967, "The "Mental' and the 'Physical", en Herbert Feigl, Michael Scriven y Grover Maxwell (comps.), Minnesota Studies in the Philosophy of Science, vol. II: Concepts, Theories, and the Mind-Body Problem, University of Minnesota Press, Minneapolis, 1958, pp. 3-116.

Fodor, Jerry, 1987, Psychosemantics: The Problem of Meaning in the Philosophy of Mind, MIT Press, Cambridge, Mass. [Versión en castellano: Psicosemántica. El problema del significado en la filosofía de la mente, trad. Oscar González Castán, Tecnos, Madrid, 1994.]

— 1983, The Modularity of Mind: An Essay on Faculty Psichology, MIT Press, Cambridge, Mass. [Versión en castellano: La modularidad de la mente. trad. José Manuel Igoa, Morata, Madrid, 1986.]

Hofstadter, Douglas y Daniel Dennett (comps.), 1981, The Mind's I, Bantam Books, Nueva York.

Kim, Jaegwon, 2014, "El fisicalismo no reduccionista y su problema con la causalidad mental", Ideas y valores, vol. 63, no. 155, pp. 235-259.

— 2002, "El problema mente-cuerpo tras cincuenta años", Azafea: Revista de Filosofía, vol. 4, pp. 45-63.

—. 1998, Mind in a Physical World: An Essay on the Mind-Body Problem and Mental Causation, MIT Press, Cambridge, Mass.

— 1993, "The Nonreductivist's Troubles with Mental Causation", Supervenience and Mind. Selected Philosophycal Essays, Cambridge University Press, Nueva York, pp. 336-357.

— 1992, "Multiple Realization and the Metaphysics of Reduction", Philosophy and Phenomenological Research, vol. 52, no. 1, pp. 1-26. 
Kim, Jaegwon, 1989, "The Myth of Nonreductive Materialism", Proceedings and Addresses of the American Philosophical Association, vol. 63, no. 3, pp. 31-47.

Lakoff, George y Mark Johnson, 1999, Philosophy in the Flesh. The Embodied Mind and Its Challenge to Western Thought, Basic Books, Nueva York.

Lewis, David, 1983, "Mad Pain and Martial Pain", Philosophical Papers, vol. 1, Oxford University Press, Oxford, pp. 122-130.

Merleau-Ponty, Maurice, 1985, Fenomenología de la percepción, trad. Jem Cabanes, Planeta-DeAgostini, Barcelona.

Mill, John Stuart, 1906, A System of Logic. Ratiocinative and Inductive, Longmans, Green and Co., Londres.

Morgan, C. Lloyd, 1923, Emergent Evolution, Williams and Norgate, Londres.

Place, U.T., 1956, "Is Consciousness a Brain Process?", British Journal of Psychology, vol. 7, no. 1, pp. 44-50. (Reimpreso en W.G. Lycan (comp.), Mind and Cognition, Blackwell, Oxford, 1990.)

Putnam, Hilary, 1960, "Minds and Machines", ed. Anderson Alan Ross, Prentice-Hall, Englewood Cliffs, 1964, pp. 72-97.

Smart, J.J.C., 1962, "Sensations and Brain Processes", en V.C. Chappell (comp.), The Philosophy of Mind, Englewood Cliffs, Prentice Hall, 1962, y reimpreso en D.M. Rosenthal (comp.), The Nature of Mind, Oxford University Press, Oxford, 1991, pp. 169-176.

Stoljar, David, 2010, Physicalism, Routledge, Nueva York.

Varela, F., E. Thompson, y E. Rosch, 1991, The Embodied Mind. Cognitive Science and Human Experience, MIT Press, Cambridge, Mass. [Versión en castellano: De cuerpo presente. Las ciencias cognitivas y la experiencia humana, trad. Carlos Gardini, Gedisa, Barcelona, 1992.]

Recibido el 17 de julio de 2017; revisado el 16 de mayo de 2018; aceptado el 1 de junio de 2018. 